\title{
Real Magnetic Poles (Magnetic Charges) in the Physics of Magnetism, Gravitation and Levitation
}

\author{
Robert A. Sizov* \\ Solid State Physics Institute, Moscow, Russia \\ Email: sizov.robert@gmail.com
}

Received 31 March 2015; accepted 7 July 2015; published 10 July 2015

Copyright (C) 2015 by author and Scientific Research Publishing Inc.

This work is licensed under the Creative Commons Attribution International License (CC BY). http://creativecommons.org/licenses/by/4.0/

(c) (i) Open Access

\begin{abstract}
Experiments executed by author of the present article (period 1968-1992) showed that the magnetic spinorial particles (magnetic charges) are real structural components of atoms and substance and are immediate sources of all magnetic fields in Nature. Joint orbital currents of electric and magnetic charges within atomic shells are the natural sources of gravitational field which is a vortical electromagnetic field. The vector nature of the gravitational field, in essence, is analogous to the vortical magnetic field that allows entering in the physical representations of such States of the gravitational field as paragravitation and ferrogravitation. Physical masses (atoms, substance, etc.), which emit ferrogravitational field, are repelled by sources paragravitational field, for example, from Earth. It is a manifestation of the effect of levitation, which was discovered by the author of this article. The forces of the technical levitation, which are formed by technical ferrogravitational fields, can be used in transport, lifting and space technology, energy and many other areas of human activity. The main reason that the real magnetic charges were "buried alive" in modern theoretical physics is the conditions of their confinement in the structures of atoms and substance, which is radically different from the confinement of electrons. Very negative role is played here by erroneous electromagnetic concept Maxwell, in which the magnetic field was officially deprived of their own source: magnetic pole or magnetic charge.
\end{abstract}

\section{Keywords}

Magnetic Spinorial Particles (Spinors and Antispinors), Magneton and Antimagneton, Bispinor, Physical Mass, Vortical Electromagnetic (Gravitational) Field, Paragravitation and Ferrogravitation, Levitation Effect

\footnotetext{
${ }^{*}$ At present, the author works as an individual researcher, Dr. of physical and mathematical sciences.
} 


\section{Introduction}

Magnetic charges (magnetic spinorial particles) as immediate sources of all magnetic fields and magnetic manifestations in the Nature were discovered by the author in structures of atoms and substance and were presented to the scientific society in 2001 in publication [1]. Experiments that prompted the author to study the problem of participation of real magnetic spinorial particles (magnetic charges) in structure atoms and substance were his experiments with the magnetic neutron scattering in ferrimagnetic crystals [2] (1970-1971). Detected in these experiments, a significant displacement of all density so-called magnetic moments $\mathrm{Fe}^{3+}$ ions from the nuclei served as a basis for the author's assumption of possibility structural participation of real magnetic spinorial particles in atoms and substance. It is important to note that as a result of the above-mentioned displacements of the so-called magnetic moments in the crystal lattice, ferrimagnet implemented a new type of magnetic ordering: helicoidal chain magnetic structure.

The results of numerous subsequent experiments conducted by the author showed that in the structures of the physical mass (atoms, nucleons, substance, etc.) the magnetic particles (spinors and antispinors) are present in approximately the same amount as the electric particles (see Appendix I). Magnetic spinors within atomic shells (the author calls them magnetons) are fundamental material particles which by their physical parameters are magnetic analogs of electrons, i.e. they have the values of charges, spins and measures of inertia equal to electrons. Magnetons like electrons exhibit charged with negative sign $\left(\mathrm{g}^{-}\right)$, they relate to the class of leptons and by their statistic properties to fermions (the spin equal to 1/2).

Magnetic antispinors, for example antimagnetons, are true antiparticles in relation to magnetons. They have a positive magnetic charge $\left(\mathrm{g}^{+}\right)$and relate to antileptons. By their statistic properties antimagnetons are antifermions with an antispin $(-1 / 2)$ in relation to spinors. The main physical parameters of real magnetic spinorial particles form together with electrical particles the atomic shells, see in Appendix II.

Namely magnetic bispinor (magnetic spinorial pair: magneton and the corresponding him antimagneton) under rotation in the lattice conductor will be created in the plane rotation of the well-known vortical magnetic field, which is determined by the axial vector rotH. Process of formation of the latter field is described by equation: $\mathrm{k} \operatorname{rot} \mathbf{J}_{\mathrm{g}}=\operatorname{rot} \mathbf{H}$, where $\operatorname{rot}_{\mathrm{g}}$ is the vortical current of magnetic charges and in model representation is the rotating magnetic bispinor, $\mathrm{k}$ - the proportionality factor. Role of the electrical current in this process consists solely in "organizing" the vortical motion of magnetic bispinors. As noted above this process is written in the following form: $k \mathbf{J}_{\mathrm{e}}=\operatorname{rot} \mathbf{J}_{\mathrm{g}}$, where $\mathbf{J}_{\mathrm{e}}$ is the vector of the density linear electric current. Thus, the sequence of the actual physical processes in a conductor with a constant electric current, which leads to the formation of a vortical magnetic field, can be written as: $\mathbf{J}_{\mathrm{e}}-\operatorname{rot} \mathbf{J}_{\mathrm{g}}-\operatorname{rot} \mathbf{H}$. We will notice that in Maxwell's electromagnetic (EM) concept in which real magnetic charges were ignored (unintentionally), the sequence of processes at formation of a vortical magnetic field round the conductor with electric current is represented as follows: $\mathbf{J}_{\mathrm{e}}-\operatorname{rot} \mathbf{H}$.

\section{Paragravitation, Ferrogravitation and Levitational Effect}

Real magnetic charges in the structures of substance fundamentally change existing ideas about the nature of magnetism, gravitational field, and also allow you to figure out the physics of such a widespread natural phenomena like levitation.

On the basis of his own experimental and theoretical investigations [1]-[5] the author has shown that the atomic shells consisting from electric and magnetic spinorial particles (magnetic charges)will be electromagnetic, but not purely electronic as it is generally accepted with the number of magnetic particles in the atoms approximately equal to the number electrical particles. Namely electromagnetic shells of the atoms are the natural sources (generators) of gravitation field, which in really is a vortical electromagnetic field. The elementary source of the gravitational field is a spinorial electromagnetic quasi-particle which was given to the author's name s-graviton ( $\mathrm{s}=$ source). Composition of the s-graviton: two spinors (electron and magneton) and two antispinor corresponding to them. Figuratively speaking the s-graviton is a combination of electric and magnetic bispinors rotating in antiphase on the same atomic orbit. This quasi-particle can also be represented in the form of two joint orbital currents of electric and magnetic charges coordinated phase. Model representation of the vortical orbital EM-current, i.e. s-graviton must be written in the following form: $\operatorname{rot}\left[\mathbf{J}_{\mathrm{e}}-\mathbf{J}_{\mathrm{g}}\right]$, where $\mathbf{J}_{\mathrm{e}}$ and $\mathbf{J}_{\mathrm{g}}$ vectors instantaneous electric current density electric (e) and magnetic (g) charges corresponding to their vortical (circular) flows. Then the equation of the process of gravitational field formation by s-graviton can be presented in the form:

$$
\mathrm{k} \operatorname{rot}\left[\mathbf{J}_{\mathrm{e}}-\mathbf{J}_{\mathrm{g}}\right]=\operatorname{rot}[\mathbf{E}-\mathbf{H}]
$$


where $\mathbf{E}$ and $\mathbf{H}$ are vectors of instantaneous strength of electric and magnetic fields in the composition of the vortical electromagnetic (gravitational) field. Minus signs put in the above equation of the process gravitational field formation correspond both to reciprocal antiphase of orbital currents electrical and magnetic charges so and anti-orientation of vectors of instantaneous strength of electric and magnetic fields in every point of the gravitational field. As follows from the above Equation (1), the gravitational field is a vortical electromagnetic field, which is characterized by changes in the direction strength vectors $(\mathbf{E}$ and $\mathbf{H})$ electric and magnetic fields that are equal in magnitude and opposite in direction to each point. Unlike from vortical magnetic field, every point of that is answered by one vector of instantaneous strength of $\mathbf{H}$, every point of the elementary gravitational field is answered two vectors of instantaneous strength of the fields of $\mathbf{E}$ and $\mathbf{H}$ equal by value and oriented antiparallel each other.

Vectorial-vortical analogy between magnetic (a) and gravitational (b) fields display on Figure 1.

In addition, on Figure 1 shown that the vortical magnetic field is product of the revolving magnetic bispinor or, that too, of the revolving spinorial magnetic dipole. The source of the elementary gravitational field is the s-graviton that yet can defined as dynamic EM-dibispinor, i.e. two combined bispinors (electric and magnetic) circulating in antiphase on one atomic orbit. The classical equations of processes formation magnetic and electromagnetic vortical (gravitational) fields are also down on Figure 1.

If polarization of vortical vectors $\operatorname{rot}\left[\mathbf{J}_{\mathrm{e}}-\mathbf{J}_{\mathrm{g}}\right]$ of s-gravitons is realized in the structures of physical masses (in atoms, nucleons, substance etc.) what is accompanied by polarization of vortical vectors $\operatorname{rot}[\mathbf{E}-\mathbf{H}]$, then in analogy with magnetic fields ferromagnetics, the gravitational fields being emitted by these masses can be called a ferrogravitational fields (FGF). The gravitational field formed by physical masses in the absence polarization of the vortical vectors of s-gravitons in their structures is a tensor or quasi-scalar field. And again, in analogy with magnetism, such field can to define as paragravitational field (PGF).

Note 1. A natural question arises about the possibility of joint use of vectors $\mathbf{E}$ and $\mathbf{H}$ in the Formula (1). Of course, in the absence of magnetic poles in the structure of substances these vectors are incompatible, because the electric field is polar and the magnetic-vortex. If magnetic charges are real structural components of atoms and substance, both electric and magnetic fields are implemented in two natural states: the polar fields are described respectively polar vectors $\mathbf{E}$ and $\mathbf{H}$ and the vortex fields, which describes the vortex vectors rotE and rotH. It is important to emphasize that the vectors standing under the operator "rot" are the vectors of the instantaneous strength of the vortex electric and magnetic fields at each point. In his early publications the author meant these vectors in the form of $\mathbf{E}^{\mathrm{O}}$ and $\mathbf{H}^{\mathrm{O}}$, that would not confuse them with the strength vectors corresponding static fields.

The physical masses which emit a ferrogravtational fields will push off from masses-sources a paragravitational fields, for example, from Earth, that constitute open by author present article a levitational effect [6]-[8].

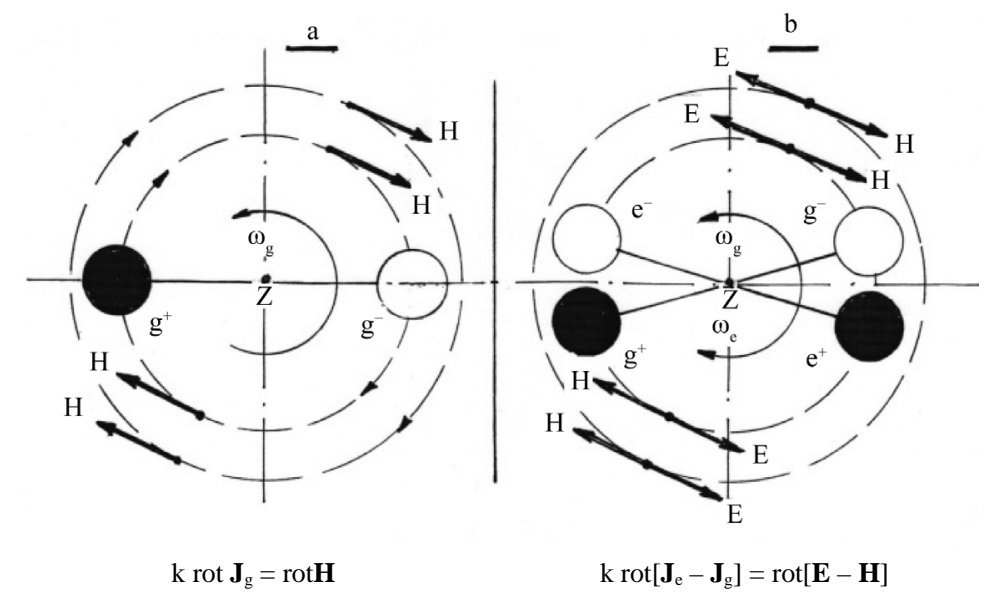

Figure 1. Schemes of mechanism the formation of the vortical fields: magnetic (a) and electromagnetic (gravitational) (b). White circles on the Figure 1 showed negative charged electric and magnetic spinors: electron $\left(\mathrm{e}^{-}\right)$and magneton ( $\mathrm{g}^{-}$) and black circles-positively charged antispinors corresponding to them with charges $\mathrm{e}^{+}$and $\mathrm{g}^{+}$. 
Such effects as volatility of the atoms (molecules) light gases, for example, hydrogen and also convectional processes in liquids and gases should be attributed to manifestations of the levitation.

The first theoretically grounded experiments in which it was received experimental gravitational field by rotating superconductors were performed by the author of this article back in 1979-1981 [1]-[3]. In the following years (1981-1991) the author received experimental gravitational field by way joint currents of magnetic and electric charges passing through static superconducting solenoid. The results of these last tests are expounded in the books of the author in Russian language [4] [5].

It's important to note that the results of our gravitational experiments are in excellent concordance with experiments of Podkletnov E. and Nieminen R. [9] and also with the experiments of Tajmar M. and de Matos C.J. [10] in which, in our opinion, the experimental gravitational field was also discovered. Unfortunately of the observed effects were explained: in work [9] as manifestation antigravitation and in the articles [10] as formation of the so called gravitomagnetic field, because their authors had no idea about presence real magnetic charges in the structural of atoms and substances.

It should be noted that definition of the gravitational field as gravitomagnetic given in [10] has relation to physical reality since both magnetic field and gravitational field are vortical fields and are described by vortical vectors: $\operatorname{rot} \mathbf{H}$ —magnetic field and $\operatorname{rot}[\mathbf{E}-\mathbf{H}]$ — gravitational field.

In his publications the author permanently underlined that without introduction of real magnetic charges into basic physical concepts it's impossible to unblock approaches for example to such important spheres of science and engineering as experimental and technical gravitation or technical levitation.

Note 2. Results of experiments author with magnetic charges and their currents in superconductors will allow him to develop technology to produce of the technical ferrogravitational field. Technical FGF and levitation forces formed by them can be used in transport, lifting and space engineering, energy, as well as in numerous gravitophysical and gravitochemical technologies ( publications author’s [7] [8]).

\section{Real Magnetic Charges in the Structure of Substance and Physical Mass}

Real magnetic charges in the structures of atoms allow us to determine the Physical mass as electromagnetic structure of atomic type, consisting of electric and magnetic charges, the main characteristic feature of which is the emission of gravitational field.

Further it's important to note that the first man who has experimentally observed magnetic charges has been $\mathbf{F}$. Ehrenhaft [11] who had published about forty articles from 1910 till 1945 devoted to their discovery and examination. Experiments Ehrenhaft are magnetic analogues of well-known experiments of Millikan to determine the charge of an electron. Very small particles of various solid substances were placed into vertical uniform magnetic field free from residual electric charges. The particles were illuminated by a concentrated light beam. The optical system made it possible to determine parameters of their movement. The basic result of the experiments of Ehrenhaft and his numerous followers consisted in directional movement of the particles along power lines of the magnetic field. By changing the field direction the direction of particles movement also changed. According to conclusions of Ehrenhaft movements of the particles observed in his experiments, he explained charged states of particles magnetic charges of different signs.

But interpretation of experimental results which was built by Ehrenhaft by analogy with behavior of electric particles in the electrostatic field doesn't appear convincing enough. Besides the values of forces in interaction being observed were comparable for example with parasitic so called radiometric forces. It is also obvious that such serious conclusions which were made by Ehrenhaft needed deeper and first of all in the sense of development of conceptions about physical parameters of magnetic charges and their place within the substance structures. In any case, the experiments Ehrenhaft and his numerous followers, despite their reliable results, have not received recognition and were forgotten.

Note 3. Research the author of this article allow you to figure the Physics and technology of occurrence of magnetic charge on the particles of a substance observed in experiments Ehrenhaft. Because magnetic charges and electric charges exist mostly in pairs + and -, can be implemented the process of separation of charges in such pairs. This process has been repeatedly implemented by the author in the process of mechanically separating the layers of the test body in the external magnetic field (Appendix I). In preparing the particles of substance in experiments Ehrenhaft were held grinding of the original solids in the Earth's magnetic field. Thus, we can expect very rare, but still, the act of separation of magnetic charges in pairs of + and - , which is manifested 
in the form of magnetic charges on the particles in the experiments Ehrenhaft.

In 1931 the question about possibility of existence magnetic poles, so called magnetic monopoles, was considered by P. Dirac [12] within the framework of quantum electrodynamics. According to conclusions of Dirac the quantum theory like classical theory allow existence of magnetic monopoles but the minimum possible magnetic charge(g) of such particles turned out to be extremely great, according to the theory-68.5e, where e is charge of an electron.

In publications of the author [5] [13] it is shown that the values of elementary magnetic charges following from the theory of Dirac, are exclusively mistaken that is defined by incorrect substitution of the magnetic field which is let out by a static magnetic pole, on a vortical magnetic field which is formed by rotating magnetic bispinor. The last circumstance was determined by the factthat the Great theorist, as well as overwhelming majority of his colleagues by profession, was under pressure of vicious EM -Maxwell's concept.

At the same time, very important position of the theory of a magnetic monopole of Dirac is the statement what exactly magnetic charges are responsible for quantization of charges electrical particles in structures of physical mass (atoms, substance and etc.). Detection by the author of the present article of magnetic spinorial particles (magnetic charges) in structures of atomic shells is simple confirmation noted above the statement of the theory Dirac in relation with a charge electron.

In the opinion of the author of the present article the investigations of F. Ehrenhaft and P. Dirac were distinctive benchmarks on the ways of discovery and investigation of magnetic charges. However, if F. Ehrenhaft and his followers had to deal with the real magnetic charges, the fictitious Dirac's theory brought to light quite exotic (restless) magnetic monopole, alien real Physics. Unfortunately, all subsequent experimental search for magnetic monopoles were based on findings from Dirac's theory, i.e., the experimenters adhered to a so-called electric technology and they tried to extract the Dirac monopoles from of substance about the same as it was customary in transactions with electric particles. It should be noted that all these numerous and costly experiments gave only negative results.

As mentioned above, in the official theoretical physics adopted a completely different point of view on the origin of the magnetic field based on the EM concept. J. C Maxwell. According to this concept, published in 1873 [14], the magnetic poles (magnetic charges) in the substance does not exist and the magnetic field is formed exclusively by the mechanical motion of electric charges.

The stationary (not dependent on time) process of the magnetic field formation under influence of the current electrons moving in the conductor is described by the so called first equation of Maxwell: $\mathrm{k} \mathbf{J}_{\mathrm{e}}=\operatorname{rot} \mathbf{H}$, where $\mathbf{J}_{\mathrm{e}}$ is the vector of the density of current electric charges, $\mathbf{H}$ is the magnetic field strength vector and $\mathrm{k}$ coefficient of proportionality. It's easy to understand that this equation is a superficial mathematical "snapshot" of the wellknow Oersted's experiment, when only external components of the physical process: electric current and vortical magnetic field are taken into consideration.

It's important to underline that the above mentioned erroneous EM-concept of Maxwell, formulated in absence of knowledge of real microprocesses proceeding in a conductor with direct electric current, turned out to be a peculiar "grave stone" on the way positive development of physical science. This "gravestone" has significantly reduced the interest of physicists in the problem of existence of real magnetic spinorial particles and also proved to be one of the main reasons of appearance of numerous false and even mystic directions in the theoretical (predominantly mathematical) physics. It should be underlined that during all the years passed, from the moment of Oersted's discovery (1820), the official physical theory couldn't yet formulate unambiguous and definitive answer to the following question: what is really a direct source of the magnetic field and what physical microprocess in a conductor with electric current is responsible for its formation. Namely with Maxwell's fallacious EM-concept has introduced portion of mysticism in physical theory. So on the one hand, the electric spinors, for example electrons, emit electric field being determined by the vector strength $\mathbf{E}$. On the other hand, the same electrons in process of their movement in the conductor within the framework of electric current, form a vortical magnetic field the strength of which is described by the axial vector rotH according to the generally adopted physical theory of Maxwell. It should be added to this that during their movement within the framework of electric current the electrons don't stop also emit their own electric field. But it's not all yet. By rotation on some atomic orbits the electrons according to the theory acquire so called magnetic moments (orbital and spin) which are responsible for all magnetic phenomena in atoms. So there arises an impression that the electric particles-especially electrons aren't simple electrospinors but some kind of electromagnetic "monsters". For example, even in the Big Encyclopedic Dictionary "Physics" (RUS edition of 1999) can read the following: "elec- 
tric charge-source electromagnetic field” or "electric field is a particular form of the electromagnetic field".

Since 2001 (publication [1]) the author try proving in all his publication, that such a structural formation as a physical mass (PM), for example: atoms or nucleons, is a dynamic spinorial EM-complex consisting of four independent fundamental particles: two spinors (electric and magnetic) and two antispinors corresponding to them. Without such a quartet the physical mass can't arise. A person with a developed of figurative thinking can imagine a physical mass like a four-wheel "cart" each wheel of which should be associated with one of the aboveindicated spinors and antispinors. But mathematical physics acknowledges only one "wheel”-electrical spinorsfrom the four above mentioned "wheels", that is onlymaterial particles with electric charges and practically doesn't take into account other three particles. Definition of mass taken from the Encyclopedic dictionary" Physics" can serve as illustration of such a state of affairs and has the following form: "mass is a physical value and one characteristics of matter determining its inertial and gravitational properties”. The results of the author's investigation have shown that the concept of real mass assumes totally different physical content.

In reality all fundamental fields - that is electric field and magnetic field are generated exclusively by corresponding poles_-spinors and initiation and use of Maxwell's EM-concept during 140 years has turned out to be the most unsuccessful outcome from the situation which took placein physics at the end XIX-th century as a result of altogether simple and regrettable fact-absence on that moment of reliable detection of real magnetic spinorial particles (magnetic charges).

It is important to note that in the Journals "Nature” and "Nature Physics" from 2008 to 2014 published a series of reports on the experimental detection of the so-called collective magnetic monopoles and their currents in “spin ice” (see, for example, [15] [16]). The effects observed in "spin ice” are defined, in our opinion, real magnetic spinorial particles: magnetons and antimagnetons. However, the authors of the above-mentioned experiments adhered to traditional views, explaining their results of the peculiar type of collective electronic states. This last interpretation, again in our opinion, is the result of indubitable influence on the minds of the vicious EM-concept Maxwell.

Note 4. The author of the present article had to hear statements of the historians of physical science that Maxwell himself had nothing against magnetic poles (charges) and had even intentionally left place for them in his EM-theory. But this "place” had no influence upon negative consequences which followed for this theory. Of course we can assume that the great physicist had some doubts and even could consider his EM-concept as the first approach to reality. But the subsequent generations of theorists not burden yourself with doubts and took a defective concept of Maxwell as the ultimate truth.

\section{Main Reasons which Detain Recognition of the Real Magnetic Charges in Atoms and Substance}

Here arise a natural question-how of magnetons and in general of magnetic spinorial particles, which are present in atoms and substance approximately in the same amounts what and of electric particles, succeeded remain unnoticeable during more one hundred years?

There are several reasons of this.

1) At first stage happened superficial interpretation of experimental results Oersted when were taken into account only the explicit participants of physical process: the electric current and the magnetic field around the conductor. This interpretation, ignoring the popular wisdom that "the truth never lies on the surface," took Maxwell, putting it as the basis of their EM theory that more than on one hundred years cemented mistaken view of magnetism as an emanation electricity. And all these years vicious EM-concept of Maxwell remained of the "fulcrum" on which form interpretations of the various magnetic and electromagnetic effects, i.e. in theoretical analysis of electric charges dealt solely with complete disregard for the actually existing in the atoms and substance of the magnetic charges.

2) The second and main reason-different nature of confinement electrons and magnetic spinorial particles within physical masses (atoms, substances and so on) what determined peculiarities of the zone structure of solid bodies with electric and magnetic charges as noted in the author's publications [7] [14]. Basing on his own experiments and also on the results of other researches the author has discovered that magnetons (as and antimagnetons) can't be torn out of the substances by pumping it with energy. With an increase in the internal energy of the body (target) increase the coupling strength (strength of confinement) between the magnetic charges and substance. The concept of free particle for electrical and magnetic spinorial particles of fundamentally different. 


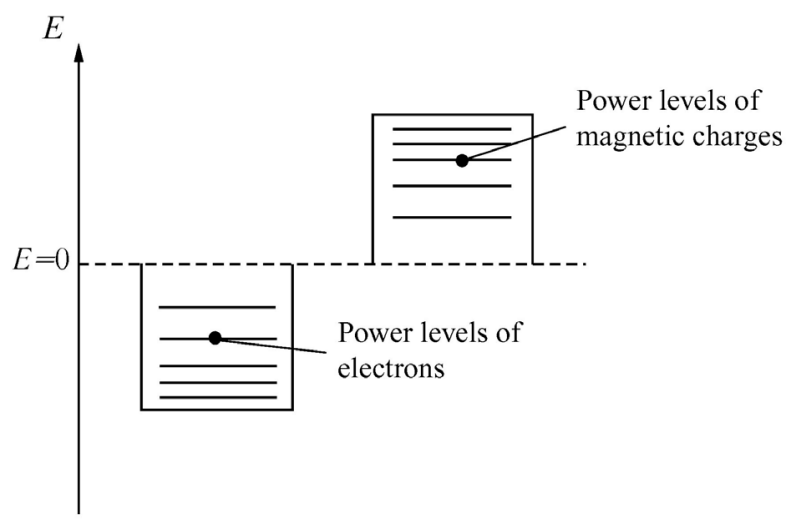

Figure 2. Location on the scale of the energy levels of the electrons (in the potential "pit") and magnetic charges (under potential “cupola”).

When an electric spinor, for example an electron, can be relatively easily extracted from the substance and can to fly freely into space, magnetons can't to leave the substance. The essence of "freedom" magnetic charges is that they, currently, do not interact with electrical particles within the substance. Free condition of magnetic poles is achieved by deep cooling of the substance (superconducting state). It's important to underline that only a part of magnetic spinorial particles occupying the so called a potential zones of conductivety can go into such a free state. It follows from the above given considerations, unlike electric spinors, it's impossible to extract an individual magnetic particle from the substance and to force it to fly through the space (outside a physical mass).

Let us explain the latter statement.

On Figure 2 shows a diagram of the energy levels of electrons and magnetons in the condensed state of substance on the scale of energy. It is well known that the electronic levels are in a potential pit and for going out of an electron of the free state, it is necessary to increase of the internal energy substance, for example, to heat him.

As for magnetons, then their levels are under potential cupola and for release their in a free condition, the substance necessary to cool, i.e. reduce its internal energy. The deep stages of cooling there arises the superconductivity effect what probably is an evidence of transition of the magnetic spinorial particles into the condition free from bonds with the substance lattice. At the same time, electric current passes through a superconductor without resistance, as the free magnetic charges revolving around the current lines do not experience FRIC of lattice conductor. Any pumping energy of the substance (radiation, heat, etc.) will push magneton to a higher energy level.

General physical conditions of confinement of spinorial particles and magnetic charges in particular within substance composition were considered in publication of the author (see [6] [7]) and the most detailed analysis was given in [13].

3) To the above mentioned causes else subjective reason should be added. In the beginning XX-th century when F. Ehrenhaft began publishing the results of their experiments related to discovery and investigations of magnetic charges the problem of these particles already not considered as sufficiently important and attractive by the majority of theoreticians. In 1931 P. Dirac again has paid attention to this problem but as was shown in the publications of the author this "shot" of the Great theoretician turned out to be "the blank" since a giant charge of the Dirac's monopole (the minimum value-68.5e) eliminates any possibilities of participation of this monopoles in the structures of physical masses (atoms, substance etc.). For convinced "electricians" the real magnetic charges turned out to be not only redundant but also in some sense harmful since their existence meant crash of "castles in the air" educated without their participation with the help of mathematical constructions.

\section{Conclusions}

The problems raised in present articles are very important for both fundamental physics and natural sciences in general. Presented by the author experimental results and conclusions based on them are brand new and require a revision of many important provisions of modern physical theory.

Experiments and theoretical investigations performed by author in Moscow from 1968 till 2013 allowed him 
to state the following:

-Magnetic field like electric field is a fundamental spinor field which is formed by its own poles—magnetic charges;

-Atomic shell, consisting of electric and magnetic spinor particles are electromagnetic (EM) but not pure electron shells, as it has always been considered;

-Just EM-shells of atoms are generators of the gravitational field which has electromagnetic nature and is a vortical of the electromagnetic field;

-Vector nature of the gravitational field similar vortical magnetic field that allows entering in the physical representations of such states of the gravitational field, as a paragravitation and ferrogravitation;

-Physical masses are electromagnetic structures of atomic type, consisting of electric and magnetic spinor particles;

-Physical masses (atoms, substance, etc.) emitting ferrogravitation field are repelled from sources parogravitation field, for example, from Earth, what is the levitation effect, discovered by the author of the article;

-Technical levitation force produced by sources (generators) ferrogravitation field, can be used in transport, lifting and space technology, energy and many other areas of human practice.

The main reasons of more than one hundred years' delay in acknowledgement and technically used magnetic charges are fundamental differences in the physics confinement of magnetic and electric particles inside atoms and substance and also the fallacious (primitive-superficial) EM-conception of Maxwell. In the framework of this concept Maxwell, in absence of real knowledge of physical processes occurring in the conductor with electric current, the strong-willed decision deprived a magnetic field own source-magnetic pole (magnetic charge). In many respects, namely "thanks to" fictitious Maxwell's electromagnetic concept, magnetic charges were "alive buried" and the magnetic field let out by them turned into electricity "abortion".

It should be added that the mere detection of magnetic spinorial particles (of magnetic charges), which managed to make the author of this article not be seen as something unique. Merit of the author is only in the fact that he showed participation of the real magnetic charges in the structures of atoms, substance and, in general, in the compositions of the physical mass. Of course, this is a very important conclusion. This was followed by a chain, in general, overarching general physical findings and experiments that, in the end, and led the author to his gravitoPhysics and gravtoTechnics. As for the fact of detection of magnetic charges, it is a merit, first of all, the wonderful Felix Ehrenhaft, who made his discoveries in the early 20th century, as well as his numerous followers [11]. Reasons which prevented the recognition of the fundamental conclusions F. Ehrenhaft, are discussed in detail in the publications of the author, and briefly discussed in this article.

\section{Acknowledgements}

The author is Grateful to technical specialists: Davydov A.A., Koshelev N.V., Lomakin A.I., Moiseenkov E.V. and Staritsin S.E. which provide technical support for the main part of his experiments, concerned the researches of magnetic charges.

\section{References}

[1] Sizov, R.A. (2001) New Presentation of Nature Magnetism, Gravitation and Nuclear Forces of Bonding (Experiments and Theory). Akademizdat Center "Science", Moscow.

[2] Sizov, R.A. (1971) Journal of Experimental and Theoretical Physics, 60, 1363-1370.

[3] Sizov, R.A. (2005) Magnetic Elementary Particles as Stable Structural Components of Atoms and Substance (Experiments and Theory). Akademizdat Center "Science", Moscow.

[4] Sizov, R.A. (2007) Magnetic Fundamental Particles (Magnetons) in Physics of Magnetic and Electromagnetic Effects (Experiments and Theory). Akademizdat Center "Science”, Moscow.

[5] Sizov, R.A. (2008) Electric and Magnetic Spinorial Particles as Structure-Forming Components of Mass and Electromagnetic Source Gravitation (Experiments and Theory). Akademizdat Center "Science”, Moscow.

[6] Sizov, R.A. (2012) Levitation as a Spread Force Manifestation of Natural Ferrogravitation. Akademizdat Center "Science", Moscow.

[7] Sizov, R.A. (2013) Transference of People and Loads in the Terrestrial Space by Means of Technical Ferrogravitation, Akademizdat Center "Science”, Moscow. 
[8] Sizov, R.A. (2013) Generation and Technical Application of Ferrogravitation (the Collection of the Invention of the Author in Area the Gravitophysical and Gravitochemical Technologies). Akademizdat Center "Science”, Moscow.

[9] Podkletnov, E. and Nieminen, R. (1992) Physica C, 203, 441-444. http://dx.doi.org/10.1016/0921-4534(92)90055-H

[10] Tajmar, M. and de Matos, C.J. (2003) Physica C, 385, 551-554.

[11] Ehrenhaft, F. (1910) Wiener Berichte, 119, 836.

[12] Dirac, P.A.M. (1931) Proceedings of the Royal Society, A133, 60-72. http://dx.doi.org/10.1098/rspa.1931.0130

[13] Sizov, R.A. (2011/2012) Matter, Antimatter and Energo-Medium-Physical Triad of the Real World. Akademizdat Center "Science", Moscow.

[14] Maxwell, J.C. (1873) Treatise on Electricity and Magnetism. Vol. 1-2, Oxford.

[15] Castelnovo, C., Moessner, R. and Sondhi, S.L. (2008) Nature, 451, 42-45. http://dx.doi.org/10.1038/nature06433

[16] Paulsen, C., Jackson, M.J., Lhotel, E., Canals, B., Prabhakaran, D., Matsuhira, K., Giblin, S.R. and Bramwell, S.T. (2014) Nature Physics, 10, 135-139. http://dx.doi.org/10.1038/nphys2847

[17] Sizov R.A. (2009) Magnetic Particles (Magnetons)—Structural Components of Atoms and Substance, Immediate Sources of Magnetic Field. Theory and Experiments (The Report at PIERS 2009 Moscow).

[18] Sizov R.A. (2009) Electric and Magnetic Spinor Particles-The Electromagnetic Source of Gravitation, Theory and Experiments (The Report at PIERS 2009 Moscow). 


\section{Appendix I}

The list of successful experiments by the author (with a brief abstract) on the problem of real magnetic spinorial particles (magnetic charges) in atoms and substance.

1. Magnetic neutron scattering [2].

Neutron diffraction studies have shown displacement of the entire density of the magnetic moments of $\mathrm{Fe}^{3+}$ ions from nuclei in the hexagonal ferrites system $\left(\mathrm{Ba}_{1-x} \mathrm{Sr}_{\mathrm{x}}\right) \mathrm{Zn}_{2} \mathrm{Fe}_{12} \mathrm{O}_{22}$. The maximum displacement was $0.9 \pm$ $0.1 \AA$. The measured intensity of the magnetic reflections $00 \mathrm{l}$ and $\mathrm{h} 0 \mathrm{l}$, as well as their indexing system showed that in the lattice of ferrites, with high probability, formed a chain helical ordering of the magnetic moments.

2. Experiments on the separation of magnetic charges in pairs + and - in a magnetic field and magnetostatic interaction of the bodies loaded with magnetic charges [1].

In mechanical separation (mechanical removal of layers with plates of copper, nickel and cadmium) in an external magnetic field of 7 - 100 e, realized charged plates magnetic charges of opposite signs. The very fact that such a charge was set by the magnetostatic interaction between the charged plates on a highly sensitive torsion balance. The magnitude of these forces in the magnetostatic interactions are evaluated as $10^{-6}-10^{-7}$ dyne.

3. Experiments on the generation of gravitation field using rotating superconductors [1].

Reel with a closed coil winding of the lead wire rotated in a bath of liquid helium. With the accelerated of the rotation of the coil observed the effect of forces on the test body (made of tungsten and lead) placed near the coils on precision balance. The forces that have been detected in these experiments were from 50 to $100 \mathrm{mg} \pm 10$ mg. According to the author's conclusions at the accelerated rotation of the superconductor in its lattice implementing joint currents electric and magnetic charges, which manifests itself through the electromagnetic vortical (gravitational) field.

4. Experiments on creation of primary magnetomotive force (MMF), magnetic analog of EMF and direct current of magnetic charges in the superconductors [3].

The author has developed a technology for the primary enrichment of magnetic charging of lead mass after mechanical separation of magnetic charges in pairs in a magnetic field (see above [2]). Magnetic charging lead mass after enrichment exceeded its primary values about 104 times. Magnetomotive force (MMF), the magnetic analog of the EMF, which was received in a pair of bodies charging magnetic charges of opposite sign, after enrichment, allowed the author to realize the currents magnetic charges in a superconducting solenoid. Thus, around superconductor is formed the vortical electric field which describes the vortical vector rotE and the process of its formation equation is at $\mathrm{k} \mathbf{J}_{\mathrm{g}}=\operatorname{rot} \mathbf{E}$, where $\mathbf{J}_{\mathrm{g}}$ is current density vector magnetic charges. The reason for the conclusion that in the experiment are precisely the vortex electric field (rotE) served a response to this field of the polarized ferroelectric arrow from barium titanate $\left(\mathrm{BaTiO}_{3}\right)$.

\section{Appendix II}

The main physical parameters of magnetic spinorial particles forming together with electrical particles the atomic shells.

Charge: The magneton (magnetic spinor) and the antimgneton (magnetic antispinor) have a magnetic charges $\mathrm{g}$ the value of which is equal to the value of the electron charge $(g=e)$. Researches of the author showed that the greatest possibility charge of a fundamental magnetic spinor particle is equal to electron charge, i.e. $\mathrm{g}_{\max }=\mathrm{e}$.

Sign of the charges: Like an electron a magneton has a charged with the sign "minus" $\left(\mathrm{g}^{-}\right)$. Antimagneton has a positive magnetic charge $\left(\mathrm{g}^{+}\right)$.

Mass: Like an electron a magneton and antimagneton are a massless particles, because the physical mass is result of joint structural "activity" of electric and magnetic spinorial particles.Class of elementary particles: The magneton is a lepton and the antimagneton-an antilepton.

Statistical properties: The magneton is a fermion (spin equal $1 / 2$ ) and the antimagneton —an antifermion (spin equal-1/2).

Note 5. Base provisions of general physics with real magnetic charges, developed by the author, were presented at the international PIERS 2009 Moscow Conference (Progress in Electromagnetics Research Symposium [17] [18]). 